1 This is the accepted version of the article. For the final version visit

2 http://www.cell.com/trends/ecology-evolution/

3

4

\title{
Conceptual domain of the matrix in fragmented landscapes
}

\author{
Don A. Driscoll, Sam C. Banks, Philip S. Barton, David B. Lindenmayer, Annabel L. Smith
}

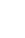

ARC Centre of Excellence for Environmental Decisions, the National Environmental Research Program Environmental Decisions Hub, Fenner School of Environment and Society, The Australian National University, Canberra ACT 0200, Australia.

In extensively modified landscapes, how the matrix is managed determines many

conservation outcomes. Recent publications revise popular conceptions of a homogeneous

and static matrix, yet we still lack an adequate conceptual model of the matrix. Here, we

identify three core effects that influence patch-dependent species, through impacts

associated with movement and dispersal, resource availability and the abiotic environment.

8 These core effects are modified by five 'dimensions': (i) spatial and (ii) temporal variation

9 in matrix quality, (iii) spatial scale, (iv) temporal scale of matrix variation, and (v)

adaptation. The conceptual domain of the matrix, defined as three core effects and their

1 interaction with the five dimensions, provides a much-needed framework to underpin

2 management of fragmented landscapes and highlights new research priorities. 


\section{A matrix focus is now both important and possible}

25 Biodiversity conservation often focusses on patches of native vegetation in a surrounding matrix

26 that is highly modified by agriculture or urbanisation $[18,19]$. The patch-matrix model of

27 landscapes [20] includes patches that are useful for conservation and the matrix in which the 28 patches are embedded [21] (see Glossary). Assumptions underpinning the patch-matrix model

29 are reasonable in many situations, particularly in fragmented and relictual landscapes where

30 there are patch-dependent species [22-24]. However, the matrix surrounding remnant vegetation

31 can have a strong influence on species occurrence and spatial dynamics $[25,26]$ and can be more

32 important than the size and spatial arrangement of remnant patches [2, 27, 28]. The growth in

33 knowledge about the matrix means it is now possible to develop a detailed synthesis of the

34 mechanisms by which the matrix directly, or indirectly drives the distribution of patch-dependent 35 species in space and time.

37 Not only is such a synthesis possible, it is also urgent. The nature of the matrix has profound

38 implications for conserving biodiversity [28, 29]. Management of the matrix can limit or

39 exacerbate the impacts of habitat loss and fragmentation [30]. Habitat loss and fragmentation are

40 the biggest threat to biodiversity globally [31]. In highly modified landscapes, further loss of

41 remnant vegetation is limited because most of it is already gone, or because what remains is

42 legally protected $[32,33]$. Where this is the case, modifying the matrix will be the major form of

43 landscape change in the future, and will therefore likely be the main process influencing

44 biodiversity conservation. There is now a pressing need for a comprehensive theoretical

45 framework of the matrix to guide the way scientists and land managers think about matrix

46 ecology. 
48 While there has been much conceptual development in the habitat fragmentation literature [22,

$4926,34]$, the concepts related to how the matrix influences patch-dependent species have not been

50 thoroughly synthesised. In this review, we build on progress made within ecological sub-

51 disciplines [25, 35, 36], and on research into edge-effects [37] and habitat fragmentation [26,

$5234]$, to describe the conceptual domain of the matrix in fragmented landscapes.

54 Our approach to understanding the conceptual domain of the matrix is to synthesise ideas from

55 the empirical literature. However, instead of providing a list of matrix effects [e.g. 25, 35, 36,

$5638,39]$, we illustrate relationships among mechanisms in a conceptual model. We demonstrate

57 through the conceptual model that what previously were considered primary effects of the matrix 58 are actually secondary outcomes of three 'core effects' (see Boxes 1 and 2). In the second part of

59 our review we identify five influential 'dimensions' and show how these modify the way that core

60 effects play out. The resulting conceptual model of the matrix can help to improve

61 communication of matrix ideas, and guide future research, including research that addresses new

62 questions about interactions between core effects and dimensions associated with time, space and 63 adaptation.

\section{Core effects of the matrix}

66 After considering the range of effects that the matrix can have on patch-dependent species [using

67 empirical literature, also canvased in numerous reviews: 19, 25, 34-36], we identified three

68 fundamental ways that the matrix influences the spatial dynamics of populations and species

69 occurrence in fragmented landscapes. The matrix can influence population persistence in 
70 fragmented systems through effects associated with (i) movement and dispersal; (ii) resource

71 availability, and; (iii) the abiotic environment (Figure 1).

72

73 Movement and Dispersal. Matrix quality influences the outcome of movement into the matrix

74 Recent reviews report that movement between patches is enhanced as the matrix becomes

75 structurally more similar to the remnant patches $[40,41]$. For example, when pastures are

76 replaced by tree plantations, colonisation of forest patches by forest specialists can increase [4].

77 However, the matrix can influence immigration and emigration in other ways. Sharp ecotonal

78 boundaries between a patch and the matrix can cause individuals to cluster inside remnants

79 ('fence effects') [1]. If a species does venture into the matrix, rapid movement through

80 unfavourable habitat could enhance connectivity between separated habitat patches [42]. On the

81 other hand, dispersal or movement between disjunct habitat patches might decline due to altered

82 behaviour, or increased mortality $[2,5,26,43]$. The influence of the matrix as a demographic

83 sink has received little research attention, although in theory, density-independent emigration can

84 increase the risk of local extinctions [44].

85

86 Resource availability. Matrix resources could aid patch-dependent species or support matrix

87 specialists.

88 The role of the matrix as a resource base for species that invade remnant patches has long been

89 understood [19] (Box 3). For example, red squirrel Tamiasciurus hudsonicus populations thrived

90 on pine-seeds in Canadian pine plantations. Squirrels subsequently invaded remnant broad-leaf

91 forest and ate Brown Creeper Certhia americana eggs, increasing the rate of nest failure of this

92 patch-dependent bird [16]. On the other hand, if the right resources are provided, the matrix can 
93 be converted to habitat and desirable native species can live throughout the landscape [e.g. 45].

94 However, if species remain patch-dependent, they might nevertheless use resources within the

95 matrix as a food subsidy [34]. With the possible exception of bees that can forage outside of the

96 nesting patch [e.g. 14], evidence that patch-dependent species gather resources outside of the

97 patch to support higher population densities inside the patch is limited [e.g. 46].

99 Abiotic environment. The matrix influences microclimate and disturbance regimes of patches.

100 The physical structure of the matrix is often different from habitat patches and can alter the

101 environmental conditions within patches [19,37], particularly when treed landscapes are cleared

102 [25]. Microclimatic changes associated with increased light and wind penetration can have far-

103 reaching effects on patch-dependent species, increasing the risk of local extinction [7, 47]. In

104 addition, species that prosper under the altered microclimate can colonise remnant vegetation

105 and drive edge-sensitive species into the remnant core [37, 48].

106

107 Changes to disturbance regimes in the matrix can also affect patch-dependent species. Larger

108 and more frequent fires can occur if there are more ignitions in the matrix [11], or when the fuel

109 structure in the matrix is changed by forest logging [11, 49] or by invasive grasses [17].

110 Conversely, active fire suppression in matrix environments can reduce rates of natural

111 disturbance in patches [3]. Altered microclimate and disturbance regimes can advantage some

112 species, often invasive exotic species $[6,17]$, but disadvantage others, often species that depend

113 on remnant vegetation [8]. Increased disturbance associated with urban or mining landscapes can

114 also drive local extinctions in patches $[9,10]$. 
116 Conceptualising matrix effects as stemming from three core effects (impacts associated with

117 dispersal, resource availability, and the abiotic environment) provides a structure for identifying

118 ecological pathways that influence abundance and population survival (Figure 1). For example,

119 invasion of patches by new species has often been listed as an important effect of the matrix on

120 patch-dependent species $[19,25,35,36]$. However, our new conceptual model emphasises that

121 such colonisation can be an indirect effect of any one of the three core effects (Box 2).

122 Similarly, altered species interactions have been listed as one of four main effects of the matrix

123 [38], but these too are a consequence of the three core effects (Box 1).

124

125 Our conceptual model of core effects (Figure 1) is a substantial heuristic advance, but we think

126 there are five influential dimensions that also must be considered to define the conceptual

127 domain of the matrix. In the next section, we outline how the core effects (Figure 1) depend on

128 five modifying dimensions: (i) spatial variation in matrix quality; (ii) the spatial scale of the

129 matrix and patches; (iii) temporal variation in matrix quality; (iv) longevity and demographic

130 rates of species relative to the temporal scale of changes in the matrix, and; (v) adaptive (plastic

131 or evolutionary) responses of species (Figure 2). Patch features, including size, shape and

132 quality also influence the response of patch-dependent species to habitat loss and fragmentation

133 (Box 4). However, consideration of patch effects is beyond the scope of our review and was

134 recently examined in detail by Didham et al. [26].

135

137 Five dimensions modify how the core effects influence biodiversity

138 Spatial variation. The matrix is not spatially homogeneous 
139 Although a spatially homogeneous matrix is often assumed in metapopulation and fragmentation

140 research, many landscapes are characterised by a heterogeneous mix of land uses and habitat

141 types $[10,25,50]$. By introducing variation into dispersal patterns, the structure and quality of a

142 heterogeneous matrix can influence the degree of isolation of habitat patches [10, 27]. Matrix

143 heterogeneity might also influence the extent and symmetry of dispersal which can lead to

144 spatially-biased movement that differentially inhibits or facilitates the colonisation of particular

145 habitat patches [51, 52]. Although practical ways have been developed to explore how spatial

146 variation in matrix quality affects dispersal, empirical knowledge of matrix effects remains

147 scarce [53].

148

149 Spatial variation in matrix quality will also lead to variation in microclimate conditions, 150 imposing spatially variable edge effects $[25,54]$. Furthermore, variation in matrix quality can

151 affect taxa differently by providing contrasting resources. For example, Öckinger et al. [46]

152 found higher butterfly species richness within grassland patches surrounded by a forest matrix,

153 but higher species richness of hoverflies in grassland patches surrounded by arable land, 154 reflecting differences in food resources for these species.

157 Spatial scale. The extent of the matrix influences its impacts on patch-dependent species

158 The spatial scale of the matrix, including geographic extent and distance between patches (see

159 Glossary), has an important effect on patch-dependent species. The distance between patches is 160 well understood to influence dispersal rates [55]. Because dispersal influences the probabilities 161 of population extinction and recolonisation of patches [24], the effects of matrix scale on 
162 dispersal (i.e. longer distances between patches) can affect patch occupancy and mediate the

163 operation of patchy populations, metapopulations or isolated populations in fragmented

164 ecosystems [13].

165

166 The spatial extent of the matrix can also influence resource subsidisation and spill-over edge

167 effects, although evidence for such effects is limited. If patch-dependent species exploit

168 resources in the matrix [34], a proportionally greater area of matrix to patch could increase the

169 relative abundance of such resources. However, movement limitation and satiation can prevent

170 patch-based species from exploiting an ever-increasing amount of matrix. Spill-over of matrix-

171 specialist predators or prey into patches [56] is influenced by the scale of the matrix and patches.

172 Increasing the scale of the matrix increases the population size of matrix specialists, and can

173 cause larger spill-over edge effects [16].

174

175 The influence of the spatial extent of the matrix on the abiotic environment of patches is likely to

176 be more limited than the effects on dispersal and resources. Most edge studies disregard the

177 scale of the adjacent matrix and so understanding of such effects is rudimentary. Narrow gaps

178 like forest roads can have substantial abiotic edge effects [57]. The extent to which wider gaps

179 have bigger effects and the scale at which effects plateau is yet to be established. The extent of

180 the matrix could also influence the risk of fire, in circumstances where fires are more likely to

181 start in agricultural lands [11].

182

183 Interactions between spatial scale and spatial variation in matrix quality can have important

184 effects on populations in fragmented systems $[58,59]$. By examining the extent to which changes 
185 in population size were synchronous, Powney et al. [58] found that matrix permeability to

186 dispersal had the strongest effect on movement between patches at intermediate distances. In

187 contrast, movement between patches was relatively insensitive to matrix type at short or long

188 distances between patches. There has been limited direct study of how such interactions occur.

189 However, the effects of matrix heterogeneity are most likely to be apparent on the spatial scale of

190 individual movement behaviour [59] or the scale over which population synchrony occurs [58].

193 Temporal variation. The matrix is not static.

194 Many studies have examined dispersal through contrasting matrix types, with implications for

195 how matrix permeability is likely to change over time. For example, bird dispersal through

196 patch-matrix landscapes can increase or decline due to increases or loss of trees [60, 61].

197 However, there are few long-term studies that directly measure temporal trends in matrix use

198 through time (but see Box 3). In one example, reintroducing fire to woodland in Missouri, USA,

199 allowed collared lizards (Crotaphytus collaris) to disperse between glades and establish stable

200 metapopulations [3]. Movement through the matrix can be influenced in other ways, including

201 annual variation in crops planted in farming landscapes [62], and climatic cycles of rainfall and

202 drought $[15,63]$.

203

204 Changes in dispersal are often driven by temporal changes in resources [61, 63, 64]. Temporal

205 variation in the resource base might also lead to variation in resource subsidisation [34], but to

206 date, the limited evidence for this is largely inferential. 
208 Abiotic effects are highly dynamic [7] and change over time as a consequence of succession, 209 seasonality, and changes in species composition, management and disturbance regimes. In

210 abandoned pastures, forest can begin to re-establish, gradually reducing temperature, wind,

211 moisture and light extremes experienced at forest edges [65]. Similar changes can take place

212 seasonally in regions with distinct dry and wet seasons [66] or during droughts [67]. In addition,

213 fire regimes change to become more extreme as exotic grasses invade new areas [68].

216 Temporal scale. Demographic and dispersal rates influence responses to changes in the matrix

217 Dispersal rate is a key trait determining the ability of species to exploit changes in the matrix

218 [69]. For example, in poorly dispersing lichen species, forest succession through plantation

219 harvest cycles can be too rapid for colonisation, particularly when the matrix is extensive [70].

220 Strong dispersers are in the best position to exploit short-term changes in matrix resources [71],

221 while species with intermediate dispersal abilities could benefit most from longer-lasting

222 temporal changes such as revegetation [69].

224 The ability to exploit resource pulses in the matrix also depends strongly on a species' life history 225 characteristics. For example, hairy-footed gerbils Gerbillurus paeba of southern African 226 savannas are dependent on grasslands embedded in an inhospitable shrubby matrix that is 227 maintained by heavy grazing [15]. In years when extreme rainfall triggered unusually high grass 228 growth, gerbil abundance and reproductive output in the (former) matrix increased markedly. 229 The short generation time (3 months) and high fecundity (up to 6 young per litter) of the gerbils 230 allowed them to exploit this short-term boom in seed supply [15]. In contrast, species with a low 
231 reproductive output, fixed seasonal breeding cycles, and low population growth rates are unlikely

232 to respond strongly to pulses of food resources in the matrix [72]. Resource specialisation can

233 also influence a species' ability to respond to changing resources in the matrix. Diet generalists

234 can exploit food resource pulses better than specialists because specialisation on rare and

235 ephemeral food sources is uncommon [72]. In contrast, where resources change gradually,

236 dietary specialists can replace generalists as succession advances [73].

238 Short-term changes in the abiotic environment of patches can provide opportunities that are

239 similar to short term resource pulses, but the ability of species to exploit such changes will

240 depend on their life-history and dispersal abilities. For example, species with multiple

241 generations within a year [74] or adequate dispersal [7] are able to exploit seasonal retreats of

242 abiotic edge effects and expand the area that they occupy within a patch [66].

Adaptation. A species response to the matrix can change over time.

246 Plastic and evolutionary responses of species to the matrix are rarely considered, but have the

247 potential to influence response pathways. Behavioural and morphological plasticity that

248 increases or reduces flight is widely reported, particularly for insect species in fragmented

249 landscapes [75-77]. Increased dispersal with fragmentation is advantageous when local

250 extinction is common, but lower dispersal can be beneficial if there is low extinction risk and

251 high dispersal mortality $[75,76]$. Therefore, changes in the matrix that influence dispersal-

252 related mortality [e.g. increased desiccation risk, 62], or extinction risk within patches [e.g. 
253 changes in the matrix fire regime, 68] could apply selection pressure that drives changes in

254 dispersal through the matrix over time, or invoke a rapid plastic response.

255

256 Species can also exhibit evolutionary or plastic responses to use resources within the matrix [e.g.

257 forest dung beetles expanding through farmland by using cattle dung, 78]. Adaptive responses to

258 changes in the abiotic environment are also possible [e.g. caterpillars adapted to survive in open

259 farmland environments, 77]. Such effects, however, have not been widely investigated. Recent

260 reviews of adaptation to global change indicate that, while such adaptation does occur, much

261 remains to be learnt about the extent to which adaptation can mitigate negative effects of human-

262 induced environmental change $[75,78,79]$. We nevertheless expect that adaptation (plastic or

263 evolutionary) is an important phenomenon that influences how species respond to matrix

264 conditions. It would not be surprising for the effects of a given matrix on a species to change,

265 potentially over a small number of generations [75].

268 What can be achieved with the new conceptual model?

270 By defining the conceptual domain of the matrix (Figures 1, 2, Boxes 1, 4) and emphasising how 271 core effects can be modified by the five dimensions, important new research priorities are now

272 apparent (see Box 5 Outstanding Questions). Research addressing these questions has the

273 potential to generate novel conservation strategies and improved understanding of ecological

274 phenomena in fragmented landscapes. For example, when there is substantial spatial and

275 temporal variation in matrix quality, it might be difficult for species to adapt to matrix conditions 
276 because selection pressures will be inconsistent [80]. This sets up a conundrum because

277 managmenent recommendations to increase matrix heterogeneity [81] might also inhibit

278 adaptation to a dominant matrix type. New research is also needed to understand the interaction

279 of the temporal scale of changes in the matrix with other dimensions and core effects. For

280 example, what are the trade-offs between dispersal ability, the temporal scale of changes in the

281 matrix and the spatial extent of the matrix [70]? Related to this, do species have different

282 responses to the same kind of temporal variation in the matrix (such as those caused by La Niña

283 climate events) if those events also vary in temporal scale? Our conceptual model therefore

284 provides a framework for developing research questions that lead to conditional predictions

285 about matrix effects [82]. Combined with attempts to generalise across species by considering

286 species traits $[39,41]$ (Box 5), the framework can help to understand the circumstances in which

287 particular effects might be expected.

289 Our framework also provides a new perspective to the old question of how the matrix might be

290 manipulated to support patch-dependent species [28, 30, 83]. Previously, lists of possible

291 approaches have been proposed, such as maintaining a certain proportion of forest cover of

292 particular size [30], maintaining hedge-rows or reducing insecticide use [83]. Our conceptual

293 framework means it is now possible for researchers and land managers to think about potential

294 approaches in a structured way. What ephemeral management practices in the matrix would

295 encourage dispersal across the landscape, provide additional resources for patch-dependent

296 species, or increase the core-area of remnant patches? How extensive should a manipulation be

297 to have these benefits? Using our conceptual model as a guide will help researchers to construct 
298 and test hypotheses that consider the range of ways that the matrix influences patch-dependent

299 species.

301 Our conceptual model also enables rapid learning and an improved capacity to frame research 302 about the matrix. It brings together the key phenomena through which the matrix acts on patch-

303 dependent species; it highlights the three core effects (Figure 1), and how these effects are 304 modified by five dimensions (Figure 2). In combination with considering patch features (Box 4)

305 and species interactions (Box 1), the conceptual model provides a simple scheme for people who 306 are new to the field to quickly comprehend these critical processes in fragmented landscapes. As

307 a research planning tool, it stimulates new ways of framing hypotheses about the matrix,

308 including drawing attention to novel interactions among the dimensions and core effects (Box 5). 309

310 The matrix in agricultural and urban landscapes is changing. Changes in the amount of tree 311 cover, the prevalence of exotic plant and animal species, fire regimes and land-use intensity 312 (among others) all contribute to making the matrix more or less hostile for patch-dependent 313 species. These changes could make the conservation outlook more bleak as land use intensifies, 314 for example, but matrix changes also provide opportunities to support species in patches. We

315 trust that by defining the conceptual domain of the matrix, the opportunities and risks associated 316 with matrix management can be better identified, understood and communicated. Ultimately, an 317 improved understanding of the matrix will enable land management practices that help stem the 318 ongoing decline of biodiversity. 


\section{Acknowledgements}

322 Joern Fischer, Laura Prugh, anonymous reviewers and the editor provided valuable feedback on

323 an earlier draft of our manuscript. Thanks to Clive Hilliker who prepared the figures and Nici

324 Sweaney for her Nanangroe photograph.

325

326 


\section{Figure 1. Matrix core effects}

329 The matrix can influence species abundance, community composition and ecological processes

330 within patches of native vegetation through three core effects associated with (i) movement and

331 dispersal, (ii) resources provided within the matrix, and (iii) the abiotic environment of patches.

332 Individuals that move into the matrix can risk elevated mortality, with possible consequences for 333 immigration rates and the population size of patch-dependent species. The matrix can also alter

334 dispersal by acting as a barrier to emigration, or can promote dispersal leading to increased

335 immigration. The matrix can provide resources that allow non-patch species to breed and

336 subsequently spill over into patches. The matrix could also provide food supplementation to

337 patch-based species. Resources within the matrix can also facilitate dispersal. The matrix can

338 drive abiotic edge effects, altering moisture, light, and disturbance levels. Each of these effects

339 can have consequences for individual species, and subsequently for community composition (see

340 Box 2 for a more detailed description of some pathways and Box 1 for consideration of species

341 interactions). Numbers indicate studies listed in the references that support parts of each

342 pathway.

\section{Figure 2. Five dimensions modify matrix core effects}

345 The conceptual model of the matrix consists of the three core effects (detailed in Figure 1)

346 whereby the matrix influences patch-dependent species through effects associated with

347 movement and dispersal, resource availability, and the abiotic environment. Five dimensions

348 modify the way the core effects influence patch-matrix dynamics; temporal variation and

349 temporal scale, spatial variation and spatial scale, and adaptation. Although we portray these 
350 dimensions as stacked, this does not imply any priortity of effects (although difficult to draw,

351 these could also be imagined as overlapping spheres encompassing the core effects, like

352 electrons around an atom's nucleus). The blue arrow indicates that dimensions can act together,

353 or can interact to influence the core effects. Although we emphasise phenomena related to the

354 matrix, the importance of patch characteristics and species interactions are well established

355 (Boxes 1, 4). For simplicity we have not attempted to draw all of the likely relationships between

356 patches and the factors that influence the impact of the matrix on patch-dependent species. 
359 Figure 1

360

361

362

\section{Core effects Mechanisms through which matrix quality influences species in habitat patches}

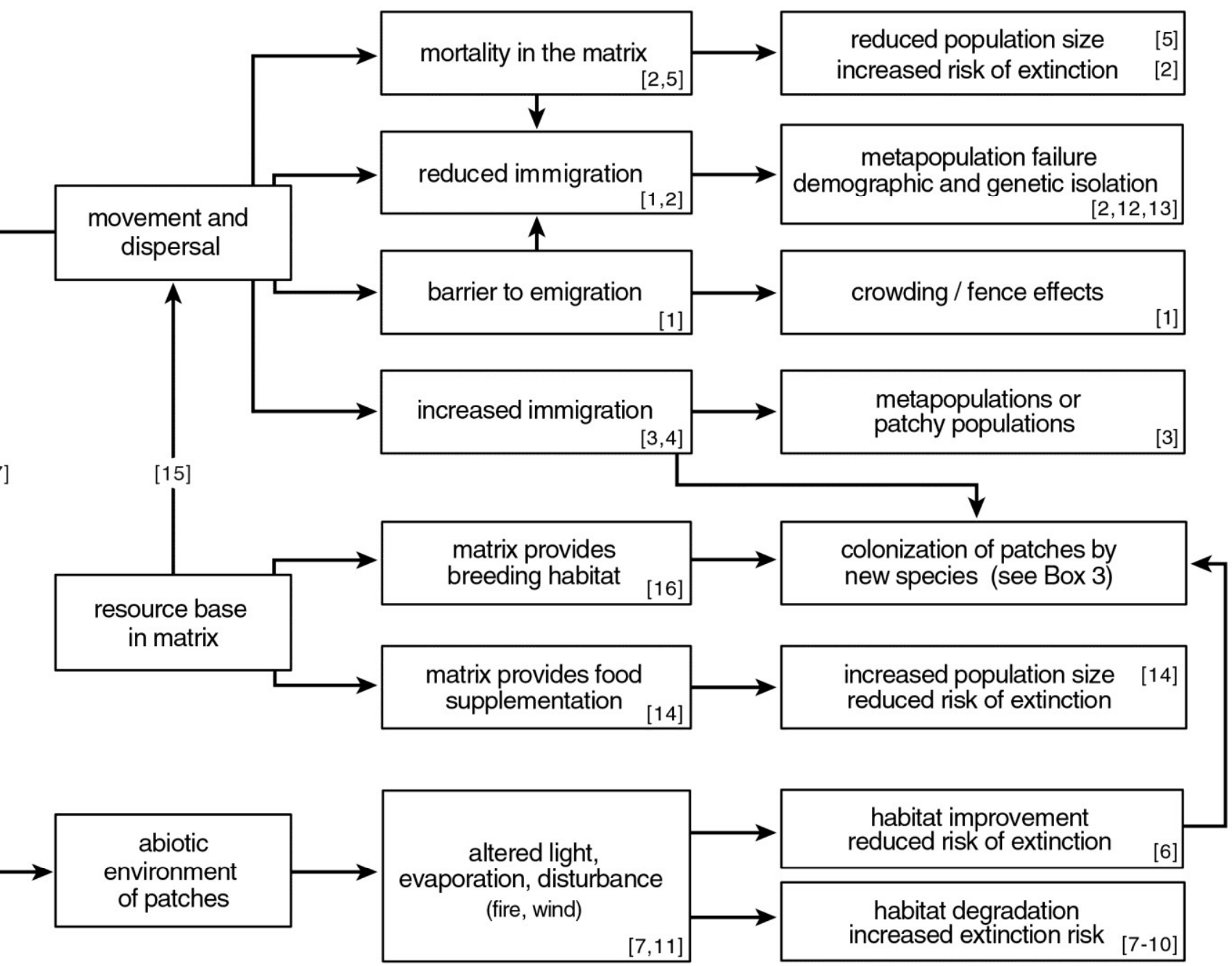


$370 \quad$ Figure 2

371

372

373

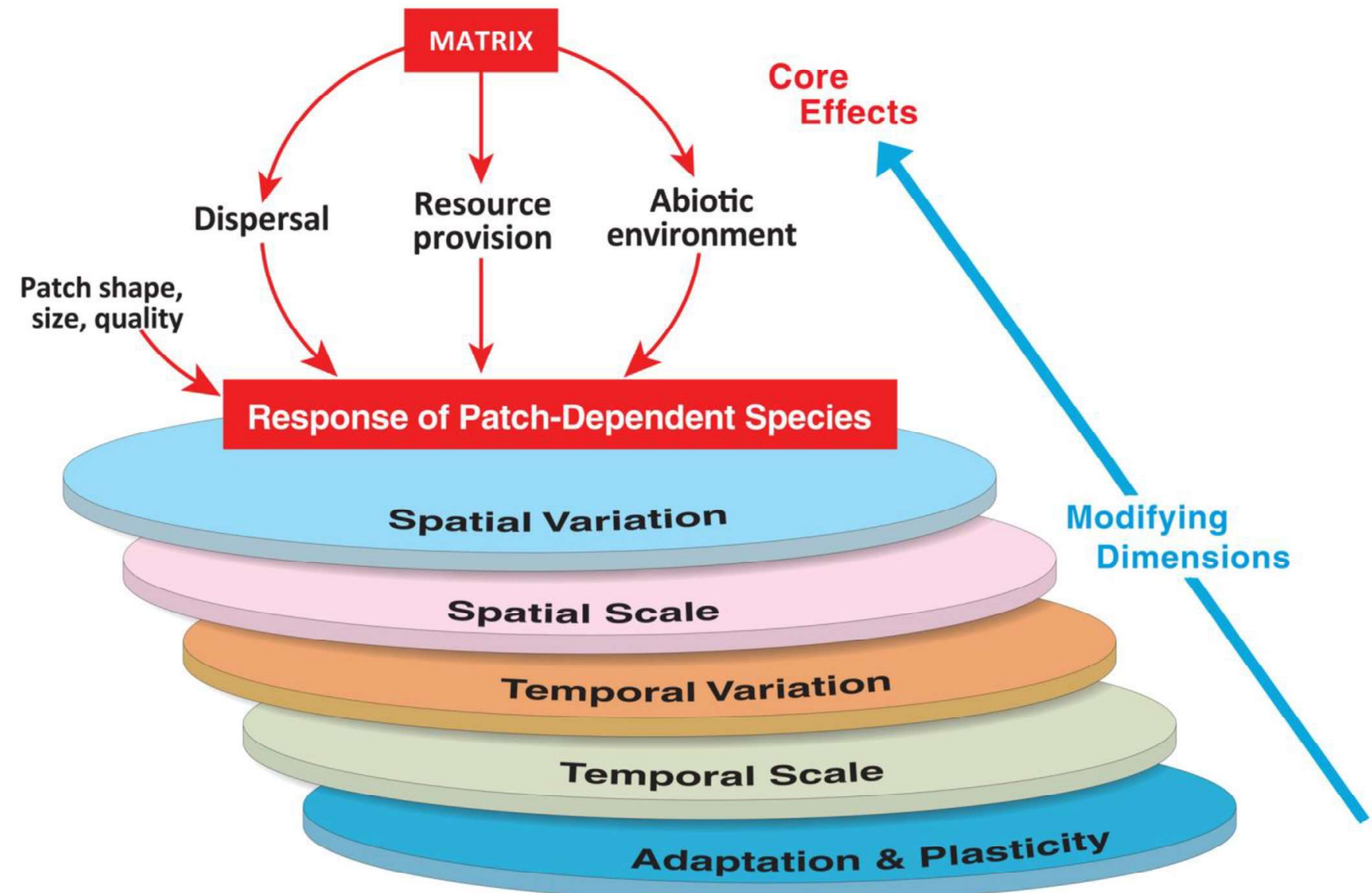




\section{Box 1. Species interactions}

377 Species interactions are integral to every step of Figure 1 (as they are to the edge-effects

378 conceptual model by Ries et al. [37] and the fragmentation conceptual model by Didham et al.

379 [26]). A pathway that affects one strongly interacting species could drive changes in many other

380 species, forming feedback loops through numerous different pathways. For example, Pita et al.

381 [2] suggested that predators can occupy degraded matrix sites in Mediterranean farmland (matrix

382 provides breeding habitat, Figure 1). The predators could inflict high dispersal mortality on

383 patch-dependent Cabrera voles Microtus cabrerae, reducing patch occupancy where the matrix is

384 highly modified. In another example, increasing resources in the matrix (seeds in wet years)

385 enabled seed-eating rodents to forage widely throughout the landscape [84]. With rodents

386 foraging beyond the patch, seed predation on hawthorn (Crataegus monogyna) within the patch

387 was reduced, providing an opportunity for recruitment of this important structural species [84].

389 Competition-colonisation trade-offs or predator-prey patch dynamics [85] might also drive

390 feedbacks between pathways in Figure 1. Where the matrix is highly permeable, a community

391 could consist of strongly competing species because poorly dispersing but competitively

392 dominant or predatory species can reach all sites. However, if the matrix offers strong resistance

393 to dispersal, the community might consist of less competitive, but strongly dispersive species

394 [86]. Our key point is that species interact. Therefore, the influence of the matrix on patch-

395 dependent species could be indirect because the matrix influences the dispersal, resources or the

396 abiotic environment of other species that depredate, out-compete or have some other interaction

397 [pollination, fruit dispersal, 64, 87] with the patch-dependent species. 


\section{Box 2. New species colonise patches by multiple pathways}

400 Invasion of patches by novel species is a widely recognised effect of the matrix on patch-

401 dependent species [25, 35, 36]. However, by defining three core effects (Figure 1), our

402 conceptual model puts colonisation of patches into a mechanistic context. Patch invasion could 403 occur through pathways that stem from each core effect.

404 1. Dispersal. A particular matrix type might allow species to disperse more effectively, increasing colonisation rates. This mechanism is supported by studies of native species becoming more prevalent in patches surrounded by a matrix suitable for dispersal. For example, the Grand Skink Oligosoma grande from New Zealand occupies rocky outcrops in either a native tussock grass matrix, or a modified pasture matrix. Higher dispersal through the native matrix contributes to a more than doubling of patch occupancy [12]. In Argentina, invasion of forest patches by the introduced Red-bellied Squirrel Callosciurus erythraeus was facilitated by structural features within the matrix such as forested strips or fences [88].

2. Resource Provision. The matrix provides resources that support a wide range of species and these can spill over into patches of native vegetation to the disadvantage of patchdependent species. For example, coffee plantations have received widespread attention as a matrix capable of supporting forest species [89], but these plantations also provide resources for pest species. In Mauritius, the Coffee Berry Moth Prophantis smaragdina moves from the matrix into adjacent rainforest, consuming the fruit and thereby reducing the reproductive success of the endemic dioecious shrub Bertiera zaluzania [90]. Such 
421 spill-over edge-effects could be more widespread than is currently recognised in the

422 literature $[56,90]$.

423

424 3. The abiotic environment. When habitat structure becomes more open and disturbed at edges of native vegetation patches, the altered abiotic conditions enables disturbancefavouring matrix species to invade patches, with consequences for patch-specialists [19, 37]. For example, in the USA, Amur Honeysuckle Lonicera maackii is a shade-intolerant invasive shrub occurring in disturbed areas and forest edges with sufficient light [91]. Invasion changed the microclimate which reduced amphibian abundance and diversity [48], along with effects on the invertebrate fauna [92]. 

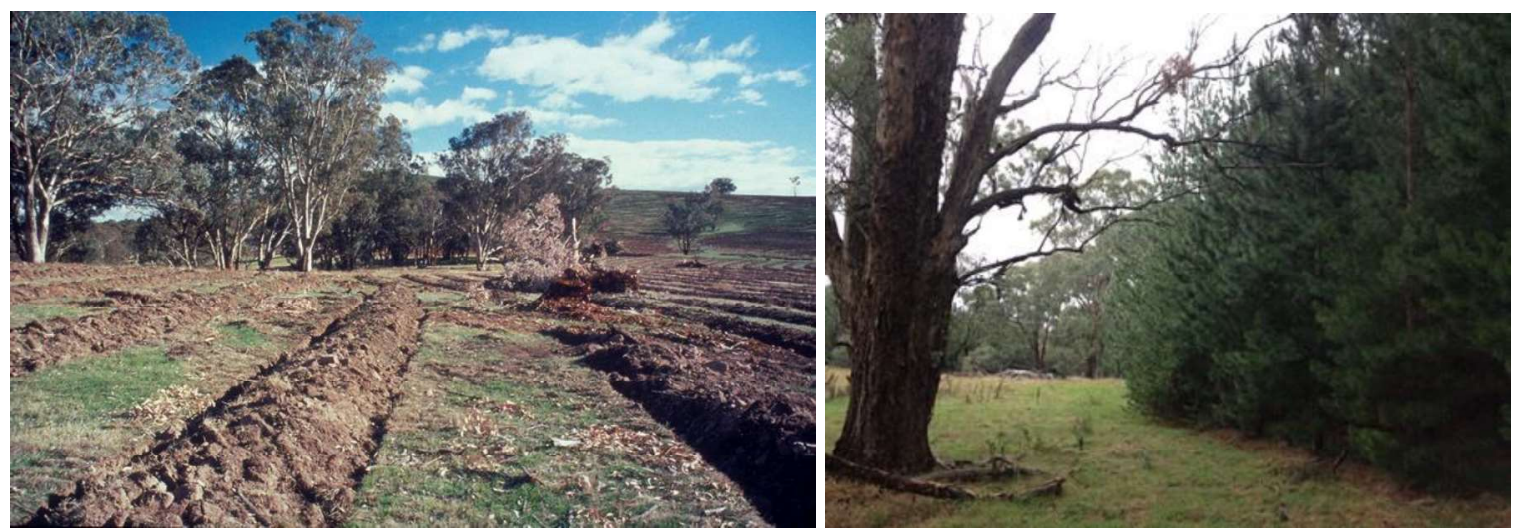

436 Figure I. A changing matrix. Pines (Pinus radiata) were planted into grazing land beginning in

437 1998. The left plate shows soil mounds scoured into the farmland in preparation for planting.

438 The trees have now grown into a dense plantation (right plate) which surrounds many remnant

439 woodland patches. The pine matrix will continue to change through cycles of thinning,

440 clearfelling and re-establishment. The dynamic matrix is likely to drive ongoing changes in the 441 animal communities of woodland patches.

444 The Nanangroe Natural Experiment was designed to quantify the effects of temporal changes in

445 the matrix on patch-dependent species in Australian temperate eucalypt woodlands [4]. The

446 major temporal change in the matrix was the transformation of a former grazing landscape into

447 one dominated by Radiata Pine (Pinus radiata) (Figure I) [93].

449 The Nanangroe study comprises 58 Eucalyptus woodland remnants surrounded by pine stands 450 and a set of 58 matched woodland "control" sites on farmland where the surrounding areas are 
451 semi-cleared grazing paddocks. The experimental design is underpinned by a randomised and

452 replicated patch selection procedure in which patches in four size classes and five woodland

453 vegetation types were identified for study [93]. Vegetation cover and selected vertebrate species

454 have been sampled on all sites every 1-2 years between 1998 and 2012, creating a high quality

455 time series dataset.

457 For birds, a range of responses to the changing matrix have been observed in the Nanangroe 458 study [4] and these illustrate some of the pathways emphasised in the conceptual model of core

459 effects (Figure 1). Key responses to the changing matrix include: (i) new species were recruited 460 to the landscape because the pine matrix provided breeding habitat (matrix provides breeding 461 habitat, Figure 1); (ii) a "spill-over" process whereby some species which increased with the 462 landscape transformation then "spilled over" from the pine matrix into adjacent woodland 463 remnants (matrix provides breeding habitat leading to colonisation of patches by new species, 464 Figure 1), and; (iii) a habitat-linked process in which some species' responses were associated 465 with measured temporal changes in vegetation attributes as the patches responded to the changed 466 abiotic conditions and management regime. For example, the ground-foraging Brown 467 Treecreeper Climacteris picumnus declined with increasing ground-level vegetation cover [4] 468 (habitat degradation leading to increased extinction risk, Figure 1). These examples underscore 469 the array of responses that can occur as a result of temporal changes in matrix quality. 


\section{Box 4. The patch still matters}

472 The matrix affects local populations through core effects associated with dispersal, the resource

473 base and the abiotic environment, but patch dynamics are also strongly influenced by

474 characteristics of the habitat patch itself. For example, does the patch offer high quality habitat

475 for a species, leading to high intrinsic growth rate or is the patch a net sink [94]? How does the

476 quality, size or shape of the patch influence the rate of emigration and immigration $[95,96]$ ?

477 How are the abiotic effects of the matrix mediated by patch shape [97]? The interaction of matrix

478 and patch effects means that the same surrounding matrix could have a large or small effect on a

479 population within a patch, depending on the species' demographic and dispersal response to

480 patch quality, size and shape.

482 The dimensions that are important modifiers of the effects of the matrix (Figure 2) might also 483 apply to patches. Habitat patches are not homogeneous and vary in quality over time [98]. The 484 rate of change of habitat quality within patches could allow, for example, long-lived species to 485 readily survive short-term changes in habitat quality [99]. Patch size is often important, but 486 spatial scale issues are more relevant when considering a matrix with multiple embedded 487 patches. Adaptation to survive in patches with altered abiotic environments, for example, might 488 also help some patch-dependent species remain in fragmented landscapes [75]. While we 489 emphasise the importance of matrix-related phenomena that influence patch-dependent species in 490 this paper, patch characteristics remain important. Whether the matrix or the patch is more 491 important for the persistence of a particular species can depend on the total amount of native 492 vegetation in the landscape, and whether the matrix or the patch is most variable. For example, if 
493 the matrix is homogeneous and relatively static, patch features might be most important, and 494 vice-versa [27].

495 
496

497

498

499

500

501

502

\section{Matrix mortality}

504 Animals that venture into the matrix can have elevated death rates [5]. In what circumstances is

505 the matrix a demographic sink and when might the sink be avoided by "fence effects" that

506 discourage movement into the matrix?

507

508

509

510

511

512 for example, are needed to solve these problems, in addition to experimental landscape

513 manipulations.

514

\section{Extent of the matrix}

516 Does the extent of the matrix influence the depth of abiotic or spill-over edge effects? If it does,

517 can the core-area of patches be increased by reducing matrix extent? 


\section{Interaction of extent and heterogeneity}

520 Are there typically lower and upper limits to the extent of the matrix beyond which there is no

521 effect of matrix quality on dispersal between patches? To explore the interaction between matrix

522 scale and heterogeneity we need improved understanding of species' dispersal limits through

523 different matrix types.

525 Interaction of extent and temporal scale

526 How does dispersal limit a species' ability to exploit matrix resources when the resources are 527 temporary [70]? For example, when an exploitable food resource becomes available in the 528 matrix, how far into the matrix can a patch-dependent species extend before the resource dries 529 up?

531 Adaptation and potential conflict with other management

532 In what circumstances does adaptation have an important influence on species survival in 533 extensively modified landscapes, and is adaptation hindered by measures, such as increasing 534 heterogeneity [81], that are aimed at promoting a less hostile matrix?

\section{Developing Generality}

537 Greatest progress towards answering the questions raised in this section will be made if research 538 simultaneously attempts to define the characteristics of species that have similar responses to the 539 matrix, enabling generalisation $[39,41]$. For example, if temporary resources are provided in the 540 matrix, what are the traits of patch-dependent species that successfully exploit the resources? 


\title{
542 Glossary
}

\author{
Matrix \\ The matrix is an extensive land-cover with different types of land- \\ cover embedded within it (patches). The matrix does not provide for \\ self-sustaining populations of some species, which are dependent \\ upon the patches. The matrix therefore, includes the extensive land- \\ cover types that patch-dependent species cannot sustainably live in. \\ This definition means that what is the matrix for some species, or was \\ the matrix at one time, might not be at other times [15] or for other \\ species [16]. \\ Patch Patches are embedded within the matrix, have vegetation that is \\ different from the matrix, and provide habitat for species that cannot \\ live in the matrix. A patch must be defined from the species point of \\ view, but this definition often coincides with a human point of view \\ because many species depend on native vegetation and cannot live in \\ cleared land or other matrix types. \\ Landscape A spatial area with diameter substantially exceeding the dispersal \\ distance of species of interest so that spatial dynamics among \\ populations can occur, such as among populations in separate patches. \\ In the context of human-dominated landscapes and species with \\ dispersal distances of a few hundred to a few thousand meters, a \\ landscape could reasonably be delineated as an area spanning 5-10 \\ $\mathrm{km}$.
}


Matrix scale Scale can be considered in terms of the distance between patches, and the overall extent of the matrix (that is, does the matrix (with or without embedded patches) extend for a few km or a few hundred $\mathrm{km}$ ?).

Matrix quality Defined from a species point of view, and referring to the features of the matrix that influence dispersal, resource availability and abiotic edge effects.

Edge The boundary between matrix and patch

Edge effect An increase or decline in abundance or occurrence of a species near the edge, often in response to altered environmental conditions near the edge or as a result of the spill-over of matrix-based species or other resources into patches [see 37]

Dispersal Movement of organisms across space [100] 


\section{References}

5471 Schtickzelle, N. and Baguette, M. (2003) Behavioural responses to habitat patch boundaries

548 restrict dispersal and generate emigration-patch area relationships in fragmented landscapes.

$549 \quad$ J. Anim. Ecol. 72, 533-545

5502 Pita, R., et al. (2007) Spatial population structure of the Cabrera vole in Mediterranean

551 farmland: The relative role of patch and matrix effects. Biol. Conserv. 134, 383-392

5523 Templeton, A.R., et al. (2011) The transition from isolated patches to a metapopulation in the 553 eastern collared lizard in response to prescribed fires. Ecology 92, 1736-1747

5544 Lindenmayer, D.B., et al. (2008) Temporal changes in vertebrates during landscape

555 transformation: a large-scale "natural experiment". Ecol. Monogr. 78, 567-590

5565 Schwab, A.C. and Zandbergen, P.A. (2011) Vehicle-related mortality and road crossing

557 behavior of the Florida panther. Appl. Geogr. 31, 859-870

5586 Keeley, J.E. (2006) Fire management impacts on invasive plants in the western United States.

559 Conserv. Biol. 20, 375-384

5607 Lehtinen, R.M., et al. (2003) Edge effects and extinction proneness in a herpetofauna from

$561 \quad$ Madagascar. Biodivers. Conserv. 12, 1357-1370

5628 Urbina-Cardona, J.N., et al. (2006) Herpetofauna diversity and microenvironment correlates

563 across a pasture-edge-interior ecotone in tropical rainforest fragments in the Los Tuxtlas

564 Biosphere Reserve of Veracruz, Mexico. Biol. Conserv. 132, 61-75

5659 Williams, N.S.G., et al. (2006) Local extinction of grassland plants: The landscape matrix is

566 more important than patch attributes. Ecology 87, 3000-3006

56710 Kennedy, C.M., et al. (2011) Landscape matrix mediates occupancy dynamics of Neotropical

$568 \quad$ avian insectivores. Ecol. Appl. 21, 1837-1850 
56911 Cochrane, M.A. and Barber, C.P. (2009) Climate change, human land use and future fires in

$570 \quad$ the Amazon. Global Change Biol. 15, 601-612

57112 Seddon, P.J., et al. (2011) Dynamics of an endangered New Zealand skink: accounting for

572 incomplete detectability in estimating patch occupancy. N. Z. J. Ecol. 35, 247-253

57313 Driscoll, D.A., et al. (2012) Spatial dynamics of the knob-tailed gecko Nephrurus stellatus in

574 a fragmented agricultural landscape. Landscape Ecol. 27, 829-841

57514 Hinners, S.J., et al. (2012) Roles of scale, matrix, and native habitat in supporting a diverse

576 suburban pollinator assemblage. Ecol. Appl. 22, 1923-1935

57715 Blaum, N. and Wichmann, M.C. (2007) Short-term transformation of matrix into hospitable

578 habitat facilitates gene flow and mitigates fragmentation. J. Anim. Ecol. 76, 1116-1127

57916 Poulin, J.-F. and Villard, M.-A. (2011) Edge effect and matrix influence on the nest survival

580 of an old forest specialist, the Brown Creeper (Certhia americana). Landscape Ecol. 26, 911-

$581 \quad 922$

58217 D'Antonio, C.M. and Vitousek, P.M. (1992) Biological Invasions by Exotic Grasses, the

583 Grass Fire Cycle, and Global Change. Annu. Rev. Ecol. Syst. 23, 63-87

58418 Lindenmayer, D.B. and Fischer, J. (2006) Habitat Fragmentation and Landscape Change. An

$585 \quad$ ecological and conservation synthesis. Island Press

58619 Saunders, D.A., et al. (1991) Biological consequences of ecosystem fragmentation: a review.

$587 \quad$ Conserv. Biol. 5, 18-32

58820 Forman, R.T.T. (1995) Land mosaics. The ecology of landscapes and regions. Cambridge

$589 \quad$ University Press

59021 Fahrig, L. and Merriam, G. (1994) Conservation of fragmented populations. Conserv. Biol. 8, $591 \quad 50-59$ 
59222 McIntyre, S. and Hobbs, R. (1999) A framework for conceptualizing human effects on

593 landscapes and its relevance to management and research models. Conserv. Biol. 13, 1282-

$594 \quad 1292$

59523 Driscoll, D.A. (2004) Extinction and outbreaks accompany fragmentation of a reptile

596 community. Ecol. Appl. 14, 220-240

59724 Hanski, I. (1999) Metapopulation Ecology. Oxford University Press

59825 Kupfer, J.A., et al. (2006) Not seeing the ocean for the islands: the mediating influence of

599 matrix-based processes on forest fragmentation effects. Global Ecol. Biogeogr. 15, 8-20

60026 Didham, R.K., et al. (2012) Rethinking the conceptual foundations of habitat fragmentation

$601 \quad$ research. Oikos $121,161-170$

60227 Bender, D.J. and Fahrig, L. (2005) Matrix structure obscures the relationship between

603 interpatch movement and patch size and isolation. Ecology 86, 1023-1033

60428 Prugh, L.R., et al. (2008) Effect of habitat area and isolation on fragmented animal

605 populations. Proc. Natl. Acad. Sci. USA. 105, 20770-20775

60629 Franklin, J.F. and Lindenmayer, D.B. (2009) Importance of matrix habitats in maintaining

607 biological diversity. Proc. Natl. Acad. Sci. USA. 106, 349-350

60830 Franklin, J.F. (1993) Preserving biodiversity - species, ecosystems, or landscapes. Ecol. Appl.

$609 \quad 3,202-205$

61031 Secretariat of the Convention on Biological Diversity (2010) Global biodiversity outlook 3.

61132 Kyle, G. and Duncan, D.H. (2012) Arresting the rate of land clearing: Change in woody

612 native vegetation cover in a changing agricultural landscape. Landscape Urban Plann. 106,

$613 \quad 165-173$ 
61433 Bradshaw, C.J.A. (2012) Little left to lose: deforestation and forest degradation in Australia

615 since European colonization. J. Plant Ecol. 5, 109-120

61634 Ewers, R.M. and Didham, R.K. (2006) Confounding factors in the detection of species

617 responses to habitat fragmentation. Biological Reviews 81, 117-142

61835 Jules, E.S. and Shahani, P. (2003) A broader ecological context to habitat fragmentation: Why

619 matrix habitat is more important than we thought. Journal of vegetation science 14, 459-464

62036 Murphy, H.T. and Lovett-Doust, J. (2004) Context and connectivity in plant metapopulations

621 and landscape mosaics: does the matrix matter? Oikos 105, 3-14

62237 Ries, L., et al. (2004) Ecological responses to habitat edges: mechanisms, models, and

623 variability explained. Annu. Rev. Ecol. Evol. Syst. 35, 491-522

62438 Campbell, R.E., et al. (2011) Production land use alters edge response functions in remnant

625 forest invertebrate communities. Ecol. Appl. 21, 3147-3161

62639 Kennedy, C.M., et al. (2010) Landscape matrix and species traits mediate responses of

627 Neotropical resident birds to forest fragmentation in Jamaica. Ecol. Monogr. 80, 651-669

62840 Eycott, A.E. (2010) Do landscape matrix features affect species movement? Systematic

629 Review CEE 08-006. Collaboration for Environmental Evidence;

$630 \quad$ http://www.environmentalevidence.org/SR43.html

63141 Prevedello, J.A. and Vieira, M.V. (2010) Does the type of matrix matter? A quantitative

632 review of the evidence. Biodivers. Conserv. 19, 1205-1223

63342 Kuefler, D., et al. (2010) The conflicting role of matrix habitats as conduits and barriers for

634 dispersal. Ecology 91, 944-950

63543 Schooley, R.L. and Wiens, J.A. (2004) Movements of cactus bugs: patch transfers, matrix

636 resistance, and edge permeability. Landscape Ecol. 19, 801-810 
63744 Hovestadt, T. and Poethke, H.J. (2006) The control of emigration and its consequences for the 638 survival of populations. Ecol. Model. 190, 443-453

63945 Karp, D.S., et al. (2011) Resilience and stability in bird guilds across tropical countryside.

$640 \quad$ Proc. Natl. Acad. Sci. USA. 108, 21134-21139

64146 Ockinger, E., et al. (2012) Landscape matrix modifies richness of plants and insects in 642 grassland fragments. Ecography 35, 259-267

64347 Ewers, R.M. and Didham, R.K. (2008) Pervasive impact of large-scale edge effects on a 644 beetle community. Proc Natl Acad Sci U S A 105, 5426 - 5429

64548 Watling, J.I., et al. (2011) Invasive shrub alters native forest amphibian communities. Biol. 646 Conserv. 144, 2597-2601

64749 Lindenmayer, B.D., et al. (2009) Effects of logging on fire regimes in moist forests. $648 \quad$ Conservation Letters 2, 271-277

64950 Ramalho, C.E. and Hobbs, R.J. (2012) Time for a change: dynamic urban ecology. Trends $650 \quad$ Ecol. Evol. 27, 179-188

65151 Gustafson, E.J. and Gardner, R.H. (1996) The effect of landscape heterogeneity on the 652 probability of patch colonization. Ecology $77,94-107$

65352 Hudgens, B.R., et al. (2012) How complex do models need to be to predict dispersal of 654 threatened species through matrix habitats? Ecol. Appl. 22, 1701-1710

65553 Zeller, K.A., et al. (2012) Estimating landscape resistance to movement: a review. Landscape $656 \quad$ Ecol. $27,777-797$

65754 Pinto, S.R.R., et al. (2010) Landscape attributes drive complex spatial microclimate 658 configuration of Brazilian Atlantic forest fragments. Trop. Conserv. Sci. 3, 389-402 
65955 Hill, J.K., et al. (1996) Effects of habitat patch size and isolation on dispersal by Hesperia 660 comma butterflies: implications for metapopulation structure. J. Anim. Ecol. 65, 725-735

66156 Rand, T.A., et al. (2006) Spillover edge effects: the dispersal of agriculturally subsidized

662 insect natural enemies into adjacent natural habitats. Ecol. Lett. 9, 603-614

66357 Delgado, J.D., et al. (2007) Edge effects of roads on temperature, light, canopy cover, and 664 canopy height in laurel and pine forests (Tenerife, Canary Islands). Landscape Urban Plann. $665 \quad 81,328-340$

66658 Powney, G.D., et al. (2011) Measuring functional connectivity using long-term monitoring 667 data. Methods Ecol. Evol. 2, 527-533

66859 Revilla, E., et al. (2004) Effects of matrix heterogeneity on animal dispersal: From individual 669 behavior to metapopulation-level parameters. Am. Nat. 164, E130-E153

67060 Tremblay, M.A. and St Clair, C.C. (2011) Permeability of a heterogeneous urban landscape to 671 the movements of forest songbirds. J. Appl. Ecol. 48, 679-688

67261 Manning, A.D., et al. (2006) Scattered trees are keystone structures - Implications for 673 conservation. Biol. Conserv. 132, 311-321

67462 Cosentino, B.J., et al. (2011) Connectivity of agroecosystems: dispersal costs can vary among $675 \quad$ crops. Landscape Ecol. 26, 371-379

67663 Blaum, N., et al. (2012) Climate induced changes in matrix suitability explain gene flow in a 677 fragmented landscape - the effect of interannual rainfall variability. Ecography 35, 650-660

67864 Magrach, A., et al. (2012) Effects of Matrix Characteristics and Interpatch Distance on

679 Functional Connectivity in Fragmented Temperate Rainforests. Conserv. Biol. 26, 238-247

68065 Laurance, W.F., et al. (2011) The fate of Amazonian forest fragments: A 32-year

681 investigation. Biol. Conserv. 144, 56-67 
68266 Hennenberg, K.J., et al. (2008) Detection of seasonal variability in microclimatic borders and 683 ecotones between forest and savanna. Basic Appl. Ecol. 9, 275-285

68467 Asbjornsen, H., et al. (2004) Synergistic responses of oak, pine and shrub seedlings to edge 685 environments and drought in a fragmented tropical highland oak forest, Oaxaca, Mexico. $686 \quad$ For. Ecol. Manage. 192, 313-334

68768 D'Antonio, C.M., et al. (2011) Long-term impacts of invasive grasses and subsequent fire in 688 seasonally dry Hawaiian woodlands. Ecol. Appl. 21, 1617-1628

68969 Donald, P.F. and Evans, A.D. (2006) Habitat connectivity and matrix restoration: the wider 690 implications of agri-environment schemes. J. Appl. Ecol. 43, 209-218

69170 Boudreault, C., et al. (2012) Epiphytic lichen colonization in regenerating black spruce forest 692 stands of clearcut origin. For. Ecol. Manage. 276, 247-258

69371 Wilcock, H.R., et al. (2007) Landscape, habitat characteristics and the genetic population 694 structure of two caddisflies. Freshwat. Biol. 52, 1907-1929

69572 Ostfeld, R.S. and Keesing, F. (2000) Pulsed resources and community dynamics of consumers 696 in terrestrial ecosystems. T.R.E.E. 15, 232-237

69773 Alanen, E.-L., et al. (2011) Differential responses of bumblebees and diurnal Lepidoptera to 698 vegetation succession in long-term set-aside. J. Appl. Ecol. 48, 1251-1259

69974 Barbosa, O. and Marquet, P.A. (2002) Effects of forest fragmentation on the beetle 700 assemblage at the relict forest of Fray Jorge, Chile. Oecologia 132, 296-306

70175 Hanski, I. (2012) Eco-evolutionary dynamics in a changing world. Ann. N. Y. Acad. Sci. 1249, $702 \quad 1-17$ 
70376 Heidinger, I.M.M., et al. (2010) Patch connectivity and sand dynamics affect dispersal-related 704 morphology of the blue-winged grasshopper Oedipoda caerulescens in coastal grey dunes.

705 Insect Conservation and Diversity 3, 205-212

70677 Merckx, T. and Van Dyck, H. (2006) Landscape structure and phenotypic plasticity in flight 707 morphology in the butterfly Pararge aegeria. Oikos 113, 226-232

70878 Tuomainen, U. and Candolin, U. (2011) Behavioural responses to human-induced 709 environmental change. Biological Reviews 86, 640-657

71079 Sih, A., et al. (2011) Evolution and behavioural responses to human-induced rapid

711 environmental change. Evolutionary Applications 4, 367-387

71280 REX Consortium (2013) Heterogeneity of selection and the evolution of resistance. Trends $713 \quad$ Ecol. Evol. 28, 110-118

71481 Shreeve, T.G. and Dennis, R.L.H. (2011) Landscape scale conservation: resources, behaviour, 715 the matrix and opportunities. J. Insect Conserv. 15, 179-188

71682 Driscoll, D.A. and Lindenmayer, B.D. (2012) Framework to improve the application of 717 theory in ecology and conservation. Ecol. Monogr. 82, 129-147

71883 Fahrig, L. (2001) How much habitat is enough? Biol. Conserv. 100, 65-74

71984 Herrera, J.M., et al. (2011) Matrix effects on plant-frugivore and plant-predator interactions in 720 forest fragments. Landscape Ecol. 26, 125-135

72185 Leibold, M.A., et al. (2004) The metacommunity concept: a framework for multi-scale 722 community ecology. Ecol. Lett. 7, 601-613

72386 Rodriguez, A., et al. (2007) Composition of an avian guild in spatially structured habitats 724 supports a competition-colonization trade-off. Proceedings of the Royal Society B-Biological $725 \quad$ Sciences 274, 1403-1411 
72687 Taki, H., et al. (2011) Plantation vs. natural forest: Matrix quality determines pollinator

727 abundance in crop fields. Sci Rep 1

72888 Bridgman, L.J., et al. (2012) Short perceptual range and yet successful invasion of a

729 fragmented landscape: the case of the red-bellied tree squirrel (Callosciurus erythraeus) in

$730 \quad$ Argentina. Landscape Ecol. 27, 633-640

73189 Philpott, S.M., et al. (2008) Biodiversity Loss in Latin American Coffee Landscapes: Review

732 of the Evidence on Ants, Birds, and Trees. Conserv. Biol. 22, 1093-1105

73390 Kaiser, C.N., et al. (2008) Exotic pest insects: another perspective on coffee and conservation.

$734 \quad$ Oryx $42,143-146$

73591 Watling, J.I. and Orrock, J.L. (2010) Measuring edge contrast using biotic criteria helps

736 define edge effects on the density of an invasive plant. Landscape Ecol. 25, 69-78

73792 McNeish, R.E., et al. (2012) Riparian forest invasion by a terrestrial shrub (Lonicera maackii)

738 impacts aquatic biota and organic matter processing in headwater streams. Biol. Invasions

$739 \quad 14,1881-1893$

74093 Lindenmayer, D.B., et al. (2001) A prospective longitudinal study of landscape matrix effects

741 on fauna in woodland remnants: experimental design and baseline data. Biol. Conserv. 101,

$742 \quad 157-169$

74394 Sanderlin, J.S., et al. (2012) On valuing patches: estimating contributions to metapopulation

744 growth with reverse-time capture-recapture modelling. Proceedings of the Royal Society B-

$745 \quad$ Biological Sciences 279, 480-488

74695 Pavlacky, D.C., Jr., et al. (2012) Anthropogenic landscape change promotes asymmetric

747 dispersal and limits regional patch occupancy in a spatially structured bird population. $J$.

$748 \quad$ Anim. Ecol. 81, 940-952 
74996 Nams, V.O. (2012) Shape of patch edges affects edge permeability for meadow voles. Ecol.

$750 \quad$ Appl. 22, $1827-1837$

75197 Gonzalez-Moreno, P., et al. (2011) Is spatial structure the key to promote plant diversity in

752 Mediterranean forest plantations? Basic Appl. Ecol. 12, 251-259

75398 Mortelliti, A., et al. (2010) The role of habitat quality in fragmented landscapes: a conceptual

754 overview and prospectus for future research. Oecologia 163, 535-547

75599 Williams, D.A., et al. (2007) Genetic diversity and spatial structure of a keystone species in 756 fragmented pine rockland habitat. Biol. Conserv. 138, 256-268

757100 Vellend, M. (2010) Conceptual synthesis in community ecology. Q. Rev. Biol. 85, 183-206 